\title{
Immediately Restored Fixed Full-arch Prosthesis on Implants Placed in Both Fresh and Healed Sockets by Means of Flat-one-bridge Technique. A 7-year Retrospective Study. Running Head: Prosthetic Passivation of Implants in Full-arch Bridges
}

Enrica Giammarinaro ( $\nabla$ e.giammarinaro@gmail.com )

DDS, Fellow Researcher at Tuscan Dental Institute, Foundation for Dental Clinic, Versilia General Hospital, Lido di Camaiore (LU)

\section{Simone Marconcini}

Responsible for Research at Tuscan Dental Institute, Foundation for Dental Clinic, Versilia General Hospital, Lido di Camaiore (LU)

\section{Ugo Covani}

UniCamillus University and Director of Tuscan Dental Institute, Foundation for Dental Clinic, Versilia General Hospital, Lido di Camaiore (LU)

\section{Andrea Mascolo}

Private Practice

Marco Del corso

Private Practice

\section{Guerino Caso}

Private Practice

\section{Research Article}

Keywords: post-extraction sockets, immediate implants, immediate loading, immediate restoration, flat abut-ment

Posted Date: July 15th, 2021

DOI: https://doi.org/10.21203/rs.3.rs-690043/v1

License: (c) (i) This work is licensed under a Creative Commons Attribution 4.0 International License. Read Full License 


\section{Abstract}

Background: Aim of this retrospective study was to document the long-term clinical efficacy of a surgicalprosthetic technique (Flat-one-Bridge) involving immediate restoration of both post-extractive and nonpost-extractive implants supporting full-arch restorations.

Methods: Implants were placed adapting the axis to the available bone. Flat definitive abutments were connected at surgery and never disconnected in order to compensate for eventual implants disparallelism. Bone grafting was performed when needed. Patients received a screw-retained provisional restoration within 48 hours of surgery and a final screw-retained prosthesis within one year.

Results: Sixty-six patients received 494 implants distributed in 75 prosthesis. The median follow-up was 86 months (range 82-168 months). Only three implants failed at the last follow-up. Implant survival was $99.6 \%$.

Conclusion: The Flat-one-Bridge prosthetic protocol is a viable procedure with excellent long-term outcomes. No difference could be observed between post-extractive and non post-extractive implants in terms of clinical success.

\section{Background}

The establishment of new predictable techniques for treating edentulous patients - or patients presenting a failing dentition - with immediate fixed full-arch restorations on dental implants has been dramatically improving the standard of care for patients, often allowing fast, cost-effective rehabilitation of the jaws [1-3].

Despite the traditional Branemark approach was meant to be a two-step treatment with implants left healing unloaded for at least 3 to 6 months, several upgrades of the procedure have been introduced, with the ultimate advent of immediate loading at post-extractive sites allowing fixed full-arch restoration of the jaws with no waiting time-frames $[4,5]$. Immediate loading protocol allows patients to wear their implantsupported prostheses within the first week after implant surgery, avoiding a secondary surgery and provisional prosthesis which are often barely functional [6].

Patients' quality of life is severely affected by tooth loss and the implant-retained prosthesis has been documented as a much more satisfying solution than removable denture in the eyes of patients presenting with a failing dentition [7]. A recent analysis suggested that there were no significant differences between immediate loading and delayed loading full-arch protocols in terms of clinical, radiological, or patient-related outcomes [8], thus meaning that, shortened procedures should always be preferred, medical indication and oral surgeon being equal. In fact, no matter how appealing it may sound, immediate implant placement (IIP) with immediate loading comes with several feasibility remarks. Contrasting local infection, achieving implant primary stability, and respecting the intended implant position represent only few of the short-range surgical tasks that directly influence the outcome of IIP. The 
relative compliance with those requirements depends primarily on the clinician expertise, as well as on given instruments and implant design [9].

High survival rates have been reported for full-arch prosthesis on implants placed in both healed and extractive sites, with no difference in terms of implant failure rates between the two situations [10]. However, most of the studies concerning full-arch restorations on failing dentitions have addressed the efficacy of computer-planned guided surgery, often not mentioning those cases where implant placement was pursued with no drilling templates [11]. It must be stressed that the survival rates of immediate implants depends on several factors coming way before the availability of a surgical guide: primary stability, implant design and dimension, surgical technique, and number of implants.

The aim of the present study was to evaluate the clinical outcome of implants placed in healed or extractive sites and then immediately loaded with full-arch restorations by means of Flat-one-Bridge technique which should allow an easy prosthetic phase and a convenient passivation of the suprastructure [12-14].

\section{Methods}

The present retrospective cohort study analyzed data retrieved from patients treated at different clinics (main center: Istituto Stomatologico Toscana, Forte dei Marmi, LU, Italy) with fixed full-arch rehabilitations on dental implants between 2007 and 2019. The investigation was performed according with the principles embodied in the Helsinki Declaration of 1964, amended in 2008, for biomedical research involving human subjects. All patients were informed about the nature of the study and gave written consent to anonymous data collection, analysis, and, eventually, scientific publication.

Patients who had previously undergone extraction of remaining failing teeth and immediate fixed-fullarch rehabilitation were considered eligible for inclusion in the present study (Fig. 1, Fig. 2). Furthermore, patients were included in the analysis if respecting the following criteria: age $\geq 18$ years, systemically healthy, compliant with adequate hygiene maintenance and follow-up visits. All patients received six or more implants positioned across the entire arcade and stabilized with insertion torque $>35 \mathrm{Ncm}$. Exclusion criteria were: presence of any local or systemic factor that might have not indicated oral surgery, poor oral hygiene, heavy smoking habits (>10/day), pregnancy, history of drug or alcohol abuse.

Data were collected regarding patient-related information (gender, age at surgery, systemic condition, smoking habit, oral hygiene), implant-related information (diameter, length, healed/extractive site, position, number per arch, tilt, loading, and finally regarding the prosthetic rehabilitation (material, complication, follow-up). Furthermore, any complication occurred during or after the surgery was taken into account and summed up to build the cumulative success and survival rate of both implants and entire rehabilitations.

\section{Surgical Procedure}


A few experienced clinicians performed the surgeries according to standard protocols. The surgical procedure was performed under local anesthesia (articaine chlorhydrate 1:1000000 adrenaline) and all patients were given $2 \mathrm{gr}$ of amoxicillin 1 hour before surgery and it was continued twice a day for 6 days thereafter (GlaxoSmithKline spa). Each patient rinsed with ozonized water for $1 \mathrm{~min}$ before and after the intervention (Aquolab srl). Before implant placement, failing teeth were extracted with a mini-invasive technique - eventually with the aid of a magneto-strictive device (Magnetic Mallet, Osteocom) - with the intent to preserve the maximum amount of residual bone. Implants were placed both in healed and in post-extractive sites, depending on the specific situation, namely wherever primary stability could be achieved. The surgeons prepared implant sites with standard drills of increasing diameter and the final implant shoulder position was meant to be bone-level or slightly sub-crestal. To ensure primary stability, the drilling protocol sometimes included under-preparation of the implant bed according to the local bone density. The longest possible implants were placed in post-extractive sites with the aim of achieving apical primary stability. Implants were inserted with a torque controller (Osstel) and avoiding excessive detrimental torque. All patients were treated with FOB over at least 6 Ossean surface implants (Intra-Lock, Boca-Raton, FL, USA) positioned across the entire arch and stabilized with insertion torque not inferior to $35 \mathrm{Ncm}$. The Ossean implants have an improved nano-rough low Calcium impregnated surface and specific designs adapted to immediate loading applications $[15,16]$. Their conic macro-geometry facilitates the achievement of primary stability and provides a documented early osseo-integration in the first weeks after surgery [17].

If it was indicated, interventions were associated with bone augmentation procedures combining corticospongious porcine grafts (GenOs, Roen, Turin, Italy) with Leukocyte and Platelet Rich Fibrin (L-PRF, IntraLock, Boca Raton, US) in order to enhance biological effects of the biomaterial with autologous GF derived from the specific hemo-component. Regeneration was manly meant as a mean to achieve pleasant soft tissue outcomes (Fig. 3a,b). A flapless approach was carried out when possible.

\section{Prosthetic Procedure}

Upon achievement of sufficient primary stability (torque $>35 \mathrm{Ncm}$ ), the clinician connected the definitive abutments to the fixtures in order to screw and adapt a fixed full-arch bridge (Flat One Bridge or FOB) within 72 hours from the surgical procedure (Fig. 4). The abutments used were the Flat Abutments (Flat Abutment, Intra-Lock, Boca Raton, FL, US). Connected to each implants, the Flat Abutments allow to compensate for problems of implant axis prior to the preparation of the implant-supported prosthesis, facilitating the passive fit of the prosthetic framework over a flat pillar. These abutments have been designed to reduce the forces that counteract a passive implant-to-casting fit, with the aim at decreasing the risk for bio-mechanical complications and at helping dissipating the forces acting on each single implant during cyclic loads even in cases of dis-parallelism among the fixtures.

The provisional prosthetic rehabilitation included a fixed bridge screwed on the Flat abutments. The provisional frameworks were mostly fabricated in $\mathrm{Cr}-\mathrm{Co}$ (72 arches) and covered by high pressed resin or composite. Only 3 cases were provisionally rehabilitated with metal-free glass-fibers frameworks covered 
with composite; 1 case was provisionalized with a braze-welded framework that was maintained also as definitive and covered with composite esthetic material.

Generally, after a minimal period of 4 months (Fig. 5), a definitive framework in Cr-Co passivated and supporting esthetic composite teeth was fabricated (Fig. 6); one case was provisionalized with a braze welded bar was maintained as definitive with its composite occlusal coverage; twelve cases were substituted with a metal framework supporting ceramics; one case was constructed in Cr-Co milled from a disk with CAM procedure.

Patients have been enrolled in a strict regimen of domiciliar hygiene instruction, motivation, and professional sessions. Dedicated soft brushes and ozone irrigation were recommended to keep the subtle virtual space between the mucosa and the rehabilitation clean.

\section{Follow-up and Outcome measures}

Patients were followed at 3-month intervals during the entire post-surgical period as this was the standard practice among the clinics involved in the study. Based on both clinical and radiographic criteria described by Buser and colleagues [18], each implant could be classified as successful or not. Criteria for implant failure were as follows: 1) persistent patient complaints; 2) peri-implant suppurative infection; 3 ) fixture mobility; 4) worsening radiolucency at the marginal bone level.

Marginal bone levels were assessed on peri-apical radiographs obtained via the long-cone paralleling technique with a loop film holder (Rinn, Dentsply Australia Pty Ltd, Pacific Hwy, St Leonards NSW 2065, Australia). Radiographs were standardized by means of individual resin bites. The distance between the implant-abutment connection and the first bone-to-implant contact ( $\mathrm{fBIC}$ ) on mesial and distal surfaces was recorded. The scale was calibrated by the width of the dental implant achieving a unique pixel $/ \mathrm{mm}$ ratio. The mean marginal bone level for each implant was computed merging mesial and distal variations. The marginal bone change was defined as the difference between the last follow-up and the baseline MBL value, with negative values denoting a loss in bone height.

The primary endpoint outcomes were:

- Implant survival (implant present in the arch, supporting the prosthetic restoration)

- Implant success (not presenting the signs defined above)

- Prosthetic success (functional prosthetic restoration, in the absence of any type of complications, either biological or mechanical)

Secondary outcomes were:

- Patients' satisfaction and quality of life

- Soft tissue stability (it was verified on digital photographs, each time by the same operator not involved in the surgery) 


\section{Statistical Analysis}

Statistical analysis was performed with R version 3.6.3 (2020-02-29) - "Holding the Windsock" (www.rproject.org/), a free software environment for statistical computing and graphics. Wilcoxon tests were used for scale variables and chi-square tests for categorical variables to assess their effect on prosthesis success. The time until failure was analyzed by Kaplan-Meier survival analysis. The effects of covariates on failure were analyzed by means of Mantel-Cox comparisons.

\section{Results}

A total of 66 consecutive patients ( 30 males and 36 females, mean age $59.4 \pm 10.1$ years, ranging from 41 to 84 years) were included in the study, 9 of them were treated bi-maxillary. In total, 75 arches were rehabilitated, 45 maxillary and 30 mandibular. A total of 13 prostheses were supported by 4 implants, 2 prostheses were supported by 5 implants, 24 prostheses were supported by 6 implants, 9 prostheses were supported by 7 implants, 22 prostheses were supported by 8 implants, 1 prosthesis was supported by 9 implants, and 4 prostheses were supported by 10 implants. Table 1 shows the demographic characteristics of the study cohort. 
Table 1

Demographics of the study population and relative chi-squared test for complication rate.

\begin{tabular}{|lll|}
\hline Variable & N O & Chi-squared test for complication \\
\hline Gender & 30 & 0.99 \\
Male & 36 & \\
Female & & \\
\hline Smoking habit & 24 & 0.56 \\
Smoker & 48 & \\
Non Smoker & & \\
\hline Age group & 37 & 0.98 \\
$<60$ & 35 & \\
$\geq 60$ & & \\
\hline Systemic condition & 2 & 0.91 \\
Anti-depressive therapy & 1 & \\
Coronary Heart Disease & 1 & \\
Diabetes & 3 & \\
Hypertension & 17 & \\
Periodontal Disease & & \\
\hline Arch & 45 & 0.71 \\
Maxilla & 30 & \\
Mandible & & \\
\hline Oral Hygiene & 43 & 0.42 \\
Good & 29 & \\
Poor & & \\
\hline
\end{tabular}

A total of 494 implants have been placed, 367 of them in post-extractive sites (74.2\%). Only 4 out of 494 implants have been loaded in a delayed fashion.

A flapless approach was used in 11 cases and a regeneration procedure was pursued in 41 arches (almost $50 \%$ of the cohort). Soft tissue appeared healthy and mucositis-free at the moment of the provisional removal and definitive restoration delivery, as well as at the last follow-up (Fig. 7). The radiological follow-up consistently confirmed a substantial preservation of peri-implant marginal bone loss for almost the entire cohort of patients (Fig. 8). All the implants were radiographically examined by 
one author alien to the treatment procedure (EG) with the OsiriX DICOM viewer (Pixmeo SARL, 266 Rue de Bernex, $\mathrm{CH}-1233$ Bernex, Switzerland). The mean marginal bone level was $+1.29 \pm 0.90 \mathrm{~mm}$ at six months. Seven years after loading, the mean marginal bone level reached $-0.32 \pm 0.50 \mathrm{~mm}$.

The function time of the immediate prosthesis ranged from 82 to 168 months, and the median time was 86 months. A total of 73 prostheses functioned for more than 7 years. A total of 56 patients still wore immediate prostheses by the end of our observation, despite having been warned that provisional prostheses are not intended for long-term use. The other patients opted to replace the immediate prosthesis with the final metal-acrylic prosthesis.

A total of 3 prostheses (4\%) experienced complications during the entire function period: each time the complication was related to the loss of one implant. However, the loss did not compromise the survival of the rehabilitation in any case. Thus, the prosthetic cumulative success rate computed with the KaplanMeier curve was $96 \%$.

The cumulative implant survival rate at 7 years was $99 \%$. A total of 3 implants failed in three different patients and one out of three failed implants was placed in a post-extractive site. All of the implant failures occurred in smoking patients, two in the mandible and one in the upper jaw. Graph 1 shows the cumulative implant survival rate stratified by smoking habits and Graph 2 shows the cumulative implant survival rate stratified by arch. Graph 3 shows the cumulative prosthesis survival curve. The cumulative prosthesis survival rate was $100 \%$ at 86 months which was the median for this study. However, all of those prosthesis exceeding 84 months of follow-up survived as well.

Except few patients necessitating repeated adjustment occlusion and night-guard fabrication, no further mechanical complications occurred.

Twenty-seven subjects had anamnestic notes resulted related to systemic conditions that did not prevent the intervention (16 patients reported periodontal anamnestic problems; 2 were in minor anti-depressive therapy; 5 were affected by hypertension; 1 had cardiovascular problems; 1 patient was diabetic; 33 subjects showed poor oral hygiene at the beginning, concerning gingival bleeding and residual calculus). Twenty-five patients were considered smokers (more than 10 cigarettes per day).

The Cox proportional hazards regression model was used to evaluate the association between complication and several possible risk indicators on the patient level. The multivariable Cox proportional hazards regression model showed that HR was always lower than 1 for all of the covariates. Thus, no significant influence could be recorded in relation to smoking habit, hygiene control, systemic condition, gender, age and so on.

\section{Discussion}

This retrospective study suggested that the rehabilitation of edentulous patients or patients with a failing dentition through full-arch immediate loading by means of the Flat-on-Bridge technique is a reliable and 
successful solution, even in the case of post-extractive implant placement. The present approach was focused on the passive fit of the prosthetic framework over the surface of flat abutments, thus correcting the emergence of the - eventually uneven - implant axis. The use of such abutments helps the clinician to exploit the residual bone whenever there is a lack of volume at post-extractive sites. Almost all rehabilitations successfully survived at a 7-year follow up visit, with only 3 prosthesis out of 75 presenting a complication related to a single implant failure: in each case, the prosthesis survival was ensured anyway.

The comparison of present data with those available in literature describes very similar success rates $[19,20]$.

In 2018, Gallucci and colleagues reviewed the evidence regarding oral rehabilitations with different combinations of implant placement and loading protocols: immediate implant placement and loading; immediate implant placement with early loading; immediate implant placement with delayed loading; late implant placement and late loading [21]. The analysis reported highest survival and success score for the combination of immediate implant placement with early loading, $98.2 \%$ and $98.7 \%-100 \%$ respectively. The authors suggested the importance of considering placement and loading time as a single denominator in the overall success analysis.

The few studies assessing the outcome of full-arch restorations supported by post-extractive implants and/or immediate and non-immediate implants are not homogenous.

In the present study, most of the implants had been placed in post-extractive sites, and no difference could be recorded between immediate implants and implants placed in healed ridge in terms of survival and success outcomes. This result is in line with previous literature on the same topic. Altintas and colleagues found that the success rate was the same for non-immediate and immediate implants (97.8\%) supporting full-arch fixed prosthesis [22]. More recently, Lerner and colleagues presented a retrospective clinical study on 110 implants (65 of them being post-extractive) supporting complete-arch fixed prosthesis with a very high success rate (98.2\%) [23].

Most of the studies included a digital planned template to guide surgery whenever there were failing teeth to be extracted and, thus, immediate implants to be placed. That was not the case of the present study, where implants have been placed without the aid of a digital-manufactured surgical template. Still, the cumulative implant survival rate at 7 years was $99 \%$, with only one failure occurring among immediate implants. It must be highlighted that immediate implants might represent a challenge to the ordinary surgeon and it has been suggested that skillfulness might influence the clinical outcome of postextractive implants [9]. That is even truer in the case of multi-implant positioning which requires careful pre-operatory diagnostic planning but also intra-operatory adaptability. In fact, primary stability is achieved simply where there is sufficient bone available: therefore, longer implants should be used as well as the maximum number of implants. 
The environment around immediate post-extractive implants is unique: from a biological perspective, it contains periodontal ligament remnants; from a mechanical perspective, peri-implant compressive strains arouse during the implant insertion; this phenomenon, coupled with heat transfer during drilling, creates a zone of apoptosis circumscribing the implant [24]. The same happens around some regions of an immediate implant, but the implant area facing the bone is less extensive. The osteogenesis in the alveolar fosse is primarily caused by blood clots around the implant, therefore, extensive blood lacunae around the implant surface will represent the initial chamber of osteogenesis [25].

The combination of the $\mathrm{FOB}$ procedure with crestal thickening obtained using graft of different biomaterials with Growth Factors and Fibrin Rich in Leukocytes and Platelet deriving from L-PRF led to optimal stabilization of final soft tissues. This aspect represents a huge difference with respect to other implant systematics: the reduced size of the Flat Abutment grants the maintaining of a close contact with the marginal periodontium.

The positive success rate of immediate implants in the preset study might be justified by the macroscopic mechanical passivation of the supra-structure mediated by the flat-to-flat connection. In fact, micro-movements, prosthetic unfitting, and unfavorable loading are among the factors that impair the healing process of immediate implants.

Furthermore, implant micro design contributes to the success of immediate implant placement and loading. The patented Ossean surface comes with particular hydrophilic properties that enhance the osteointegration in the firs 4 weeks after surgery: the fractal, nano-rough Ossean surface, in fact, has been reported to influence cellular genetic expression - or the fate of stem cells -at the nano-level, which in turn induces faster implants osteointegration [26].

The present was a retrospective study without a control group, therefore, its results may not be generalized. A further limitation of the study was represented by the lack of peri-implant mucosal inflammation data analysis. However, the long follow-up of the present study makes the outcome more reliable than other short-term studies on the same topic.

Long-term function of implant- supported full-arch immediate prosthesis usually leads to a high prosthetic complication prevalence, featuring the prosthesis fracture as the most common complication one during the first year of loading. In the present study, no prosthetic fracture was reported, confirming that the framework reinforcement (metallic or event glass-fibers reinforcement) could be the most important success key during the osseointegration period. Glass fiber-reinforced acrylic immediate prosthesis may function better in cases of removable denture in the opposing jaw. More comparative studies are need to demonstrate this postulate.

Even if the flat abutment protocol can be provided also with a lower number of supporting implants, the optimal number of implants was always considered a minimum of 6 and 8 implants in the lower and upper arch, respectively. Only future studies will be helpful to ascertain the minimum number of implants to be required for prosthetic support with this type of protocol.

Page $10 / 21$ 


\section{Conclusion}

In the case of FOB rehabilitations, this specific protocol may present some favorable ethical and social implications due to the accomplishment of a good cost-to-benefit ratio with a minimally invasive procedure because it helps the clinician to try achieving a passive reciprocal relationship between implants and it may also give excellent aesthetic results, good function with a reduced trauma to the bone, avoiding any reduction of the crest compared to other more invasive protocols.

\section{Abbreviations}

Immediate implant placement (IIP)

Leukocyte and Platelet Rich Fibrin (L-PRF)

Flat One Bridge (FOB)

\section{Declarations}

Ethics approval and consent to participate: The UniCamillus Ethical Committee has exempted the present study protocol from the need of its opinion.

Consent for publication: All patients gave consent.

Availability of data and material: All data are available.

Competing interests: There is no competing interest regarding this study.

Funding: There is no funding regarding this study.

Acknowledgment: Not applicable

\section{References}

1. Peñarrocha-Oltra $D$, Covani U, Peñarrocha $M$, Peñarrocha-Diago M. Immediate versus conventional loading with fixed full-arch prostheses in mandibles with failing dentition: a prospective controlled study. Int J Oral Maxillofac Implants. 2015;30(2):427-434. doi:10.11607/jomi.3534.

2. Peñarrocha-Oltra $D$, Covani U, Peñarrocha-Diago $M$, Peñarrocha-Diago $M$. Immediate loading with fixed full-arch prostheses in the maxilla: review of the literature. Med Oral Patol Oral Cir Bucal. 2014;19(5):e512-e517. Published 2014 Sep 1. doi:10.4317/medoral.19664

3. Gallardo YNR, da Silva-Olivio IR, Gonzaga L, Sesma N, Martin W. A Systematic Review of Clinical Outcomes on Patients Rehabilitated with Complete-Arch Fixed Implant-Supported Prostheses According to the Time of Loading. J Prosthodont. 2019;28(9):958-968. doi:10.1111/jopr.13104. 
4. Mello CC, Lemos CAA, Verri FR, Dos Santos DM, Goiato MC, Pellizzer EP. Immediate implant placement into fresh extraction sockets versus delayed implants into healed sockets: A systematic review and meta-analysis. Int J Oral Maxillofac Surg. 2017;46(9):1162-1177.

doi:10.1016/j.ijom.2017.03.016

5. Barone A, Marconcini S, Giammarinaro E, Mijiritsky E, Gelpi F, Covani U. Clinical Outcomes of Implants Placed in Extraction Sockets and Immediately Restored: A 7-Year Single-Cohort Prospective Study. Clin Implant Dent Relat Res. 2016;18(6):1103-1112. doi:10.1111/cid.12393

6. Giammarinaro E, Soto-Peñaloza D, Aizcorbe-Vicente J, Peñarrocha-Diago M, Covani U, PeñarrochaOltra D. Introduction to Immediate Loading in Implantology. In: Peñarrocha-Diago M., Covani U., Cuadrado L. (eds) Atlas of Immediate Dental Implant Loading. 2019; Springer, Cham. https://doi.org/10.1007/978-3-030-05546-2_1.

7. Thomason JM, Heydecke G, Feine JS, Ellis JS. How do patients perceive the benefit of reconstructive dentistry with regard to oral health-related quality of life and patient satisfaction? A systematic review [published correction appears in Clin Oral Implants Res. 2008 Mar;19(3):326-8]. Clin Oral Implants Res. 2007;18 Suppl 3:168-188. doi:10.1111/j.1600-0501.2007.01461.x

8. Cosola S, Marconcini S, Giammarinaro E, Poli GL, Covani U, Barone A. Oral health-related quality of life and clinical outcomes of immediately or delayed loaded implants in the rehabilitation of edentulous jaws: a retrospective comparative study. Minerva Stomatol. 2018;67(5):189-195. doi:10.23736/S0026-4970.18.04134-1

9. Barone A, Toti P, Marconcini S, Derchi G, Saverio M, Covani U. Esthetic Outcome of Implants Placed in Fresh Extraction Sockets by Clinicians with or without Experience: A Medium-Term Retrospective Evaluation. Int J Oral Maxillofac Implants. 2016;31(6):1397-1406. doi:10.11607/jomi.4646

10. Ciabattoni G, Acocella A, Sacco R. Immediately restored full arch-fixed prosthesis on implants placed in both healed and fresh extraction sockets after computer-planned flapless guided surgery. A 3-year follow-up study. Clin Implant Dent Relat Res. 2017;19(6):997-1008. doi:10.1111/cid.12550

11. Meloni SM, De Riu G, Pisano M, Tullio A. Full arch restoration with computer-assisted implant surgery and immediate loading in edentulous ridges with dental fresh extraction sockets. One year results of 10 consecutively treated patients: guided implant surgery and extraction sockets. J Maxillofac Oral Surg. 2013;12(3):321-325. doi:10.1007/s12663-012-0429-8

12. Bucci Sabattini V, Zampetti P, Del Corso M, La Grotta A, Rizzo L. Una nuova tecnica di carico immediato per il Toronto Bridge Dental Tribune It. 2006; 3:6-9.

13. Bucci-Sabattini V, Minnic A, Manfredini D, Mascolo A, Zaina F. The Flat One Bridge technique for fullarch edentulism: long term results from a prospective cohort study Poseido; 2013;1:177-185.

14. Covani U, Orlando B, D’Ambrosio A, Sabattini VB, Barone A. Immediate rehabilitation of completely edentulous jaws with fixed prostheses supported by implants placed into fresh extraction sockets and in healed sites: a 4-year clinical evaluation. Implant Dent. 2012;21(4):272-279.

doi:10.1097/ID.0b013e31825885e0 
15. Shibli JA, Grassi S, Piattelli A, et al. Histomorphometric evaluation of bioceramic molecular impregnated and dual acid-etched implant surfaces in the human posterior maxilla. Clin Implant Dent Relat Res. 2010;12(4):281-288. doi:10.1111/j.1708-8208.2009.00174.x

16. Coelho PG, Granato R, Marin C, et al. Biomechanical evaluation of endosseous implants at early implantation times: a study in dogs. J Oral Maxillofac Surg. 2010;68(7):1667-1675. doi:10.1016/j.joms.2010.02.050

17. Dohan Ehrenfest DM. Fractal patterns applied to implant surface: definitions and perspectives. J Oral Implantol. 2011;37(5):506-509. doi:10.1563/AAID-JOI-D-11-00081

18. Buser D, Weber HP, Lang NP. Tissue integration of non-submerged implants. 1-year results of a prospective study with $100 \mathrm{ITI}$ hollow-cylinder and hollow-screw implants. Clin Oral Implants Res. 1990;1(1):33-40. doi:10.1034/j.1600-0501.1990.010105.x

19. Pomares C. A retrospective study of edentulous patients rehabilitated according to the 'all-on-four' or the 'all-on-six' immediate function concept using flapless computer-guided implant surgery. Eur $J$ Oral Implantol. 2011 Summer;4(2):125.

20. Fischer K, Stenberg T. Prospective 10-year cohort study based on a randomized controlled trial (RCT) on implant-supported full-arch maxillary prostheses. Part 1: sandblasted and acid-etched implants and mucosal tissue. Clin Implant Dent Relat Res. 2012;14(6):808-815. doi:10.1111/j.17088208.2011.00389.x

21. Gallucci GO, Hamilton A, Zhou W, Buser D, Chen S. Implant placement and loading protocols in partially edentulous patients: A systematic review. Clin Oral Implants Res. 2018;29 Suppl 16:106134. doi:10.1111/clr.13276

22. Altintas NY, Taskesen F, Bagis B, Baltacioglu E, Cezairli B, Senel FC. Immediate implant placement in fresh sockets versus implant placement in healed bone for full-arch fixed prostheses with conventional loading. Int J Oral Maxillofac Surg. 2016;45(2):226-231. doi:10.1016/j.ijom.2015.09.011

23. Lerner H, Hauschild U, Sader R, Ghanaati S. Complete-arch fixed reconstruction by means of guided surgery and immediate loading: a retrospective clinical study on 12 patients with 1 year of follow-up. BMC Oral Health. 2020;20(1):15. Published 2020 Jan 16. doi:10.1186/s12903-019-0941-z

24. Yuan X, Pei X, Zhao Y, et al. Biomechanics of Immediate Postextraction Implant Osseointegration. $J$ Dent Res. 2018;97(9):987-994. doi:10.1177/0022034518765757

25. Kuzyk PR, Schemitsch EH. The basic science of peri-implant bone healing. Indian J Orthop. 2011;45(2):108-115. doi:10.4103/0019-5413.77129

26. Coelho PG, Takayama T, Yoo D, et al. Nanometer-scale features on micrometer-scale surface texturing: a bone histological, gene expression, and nanomechanical study. Bone. 2014;65:25-32. doi:10.1016/j.bone.2014.05.004

\section{Figures}




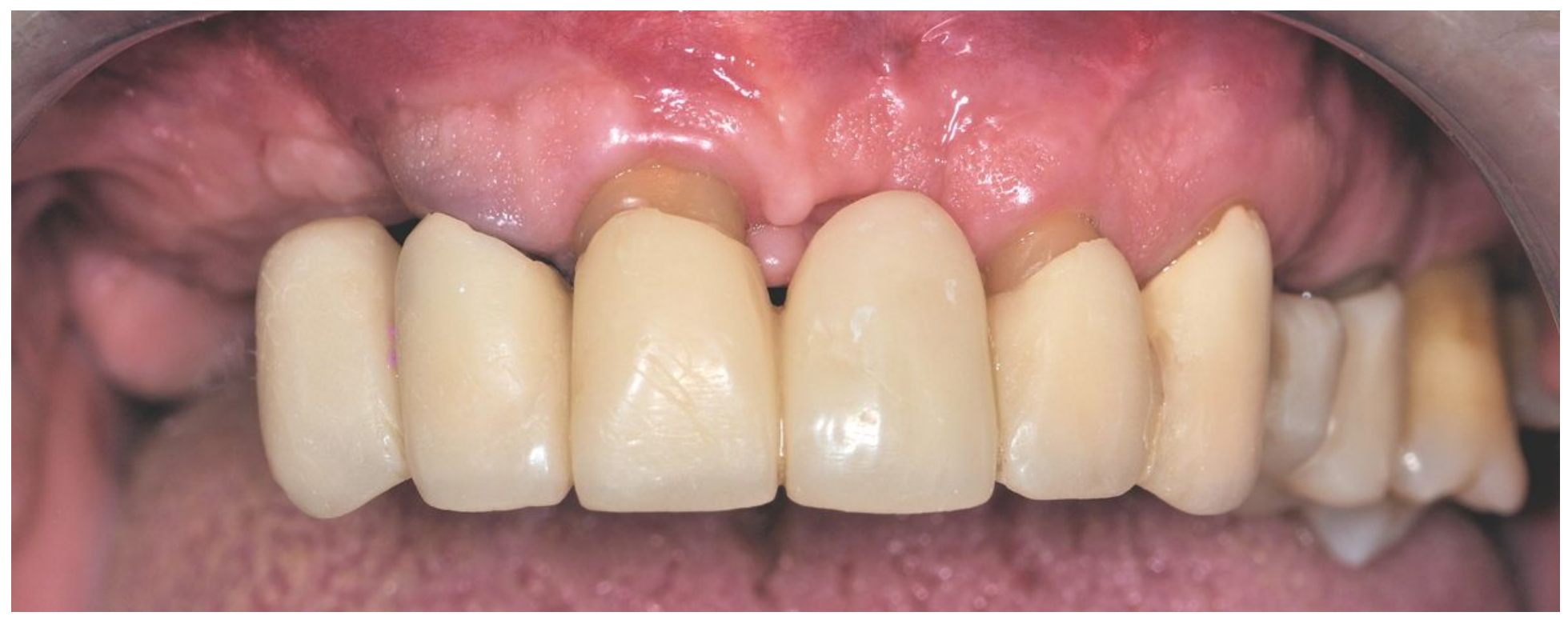

Figure 1

Intra-oral photograph of one of the male patients presenting with failing upper dentition.

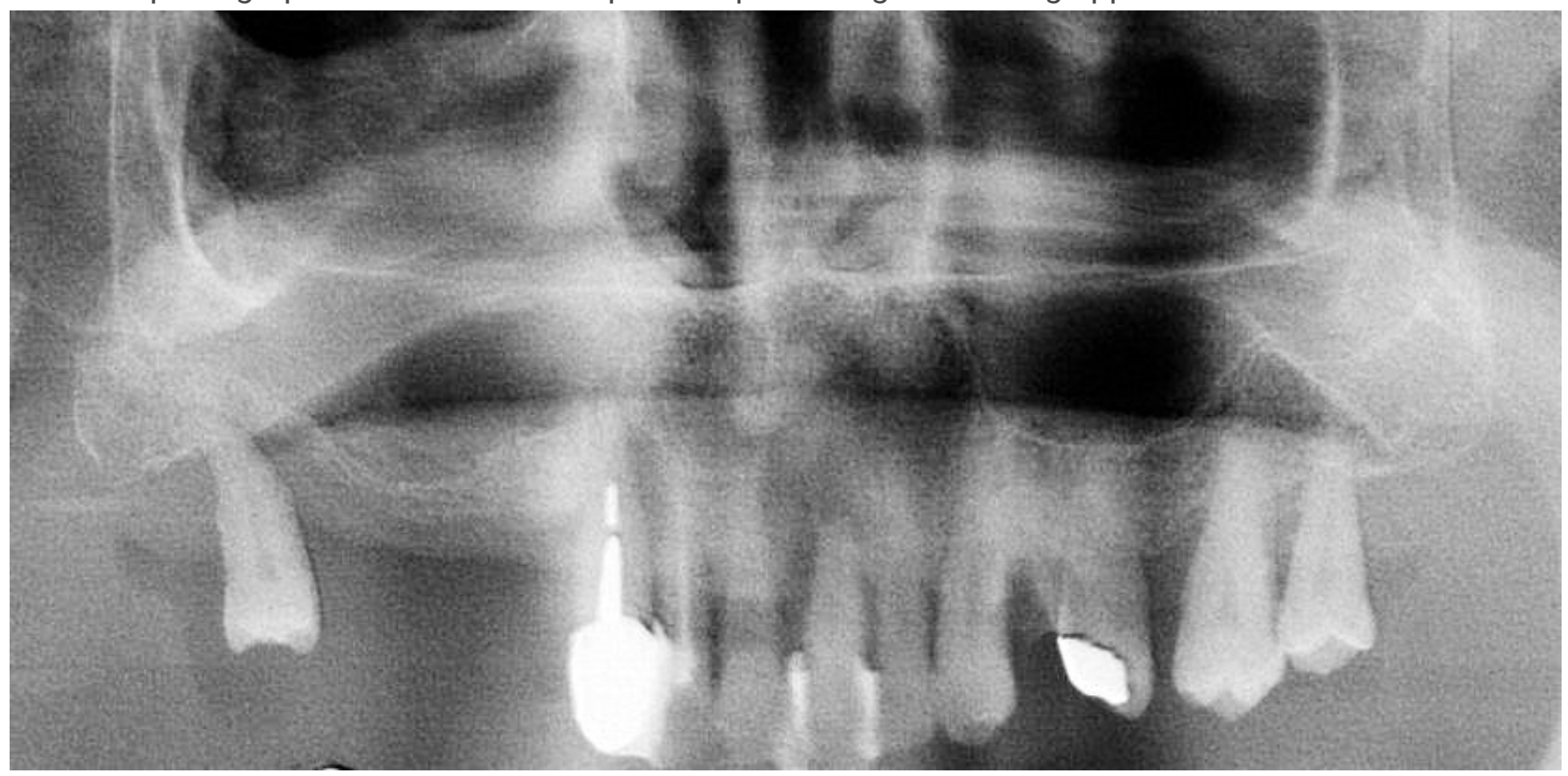

Figure 2

Panoramic radiograph of the same patient. 

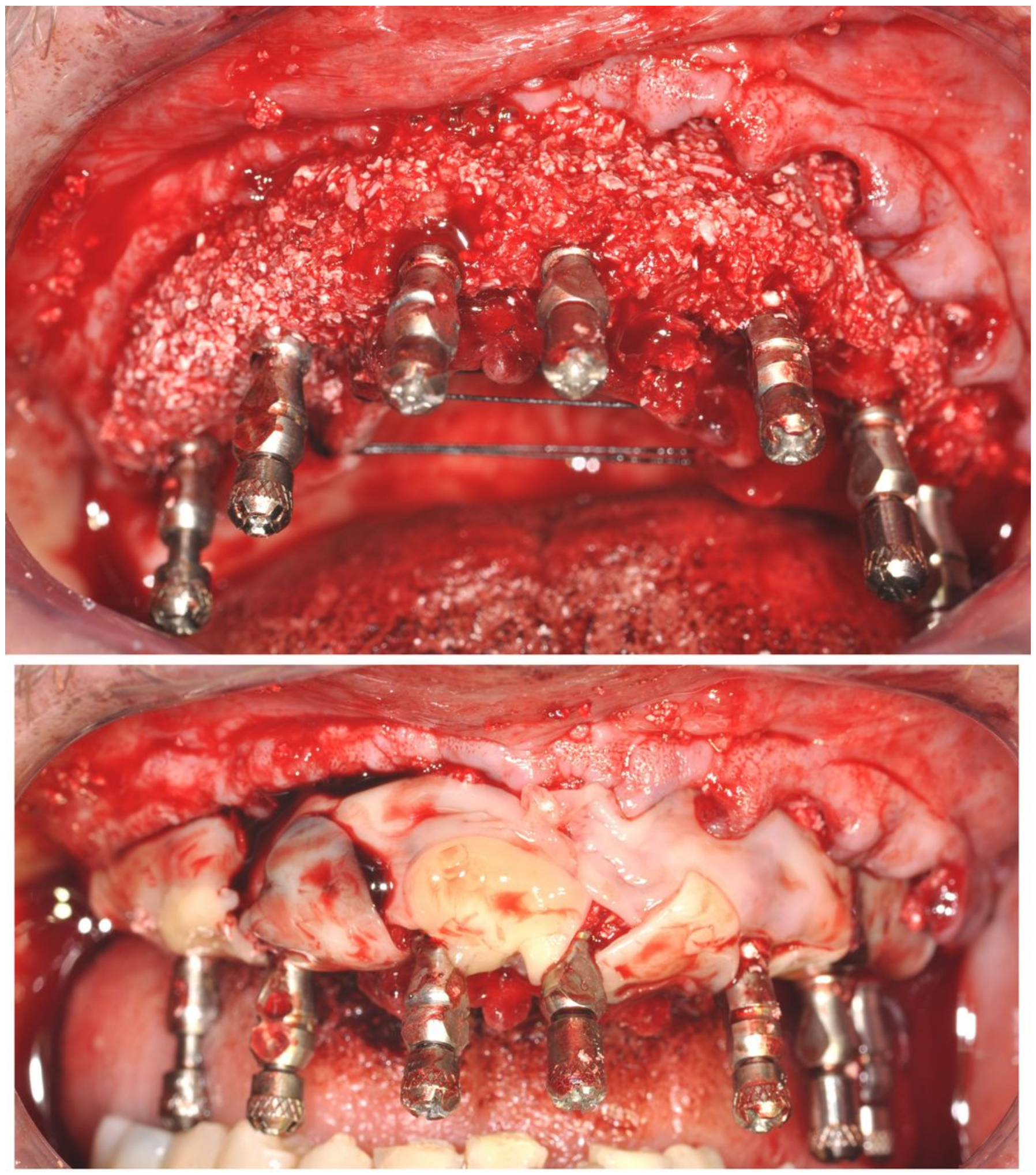

Figure 3

a. Intra-oral photograph of the upper arch right after surgery with bone graft showing. b. Intra-oral photograph of the lower arch right after surgery with L-PRF showing. 


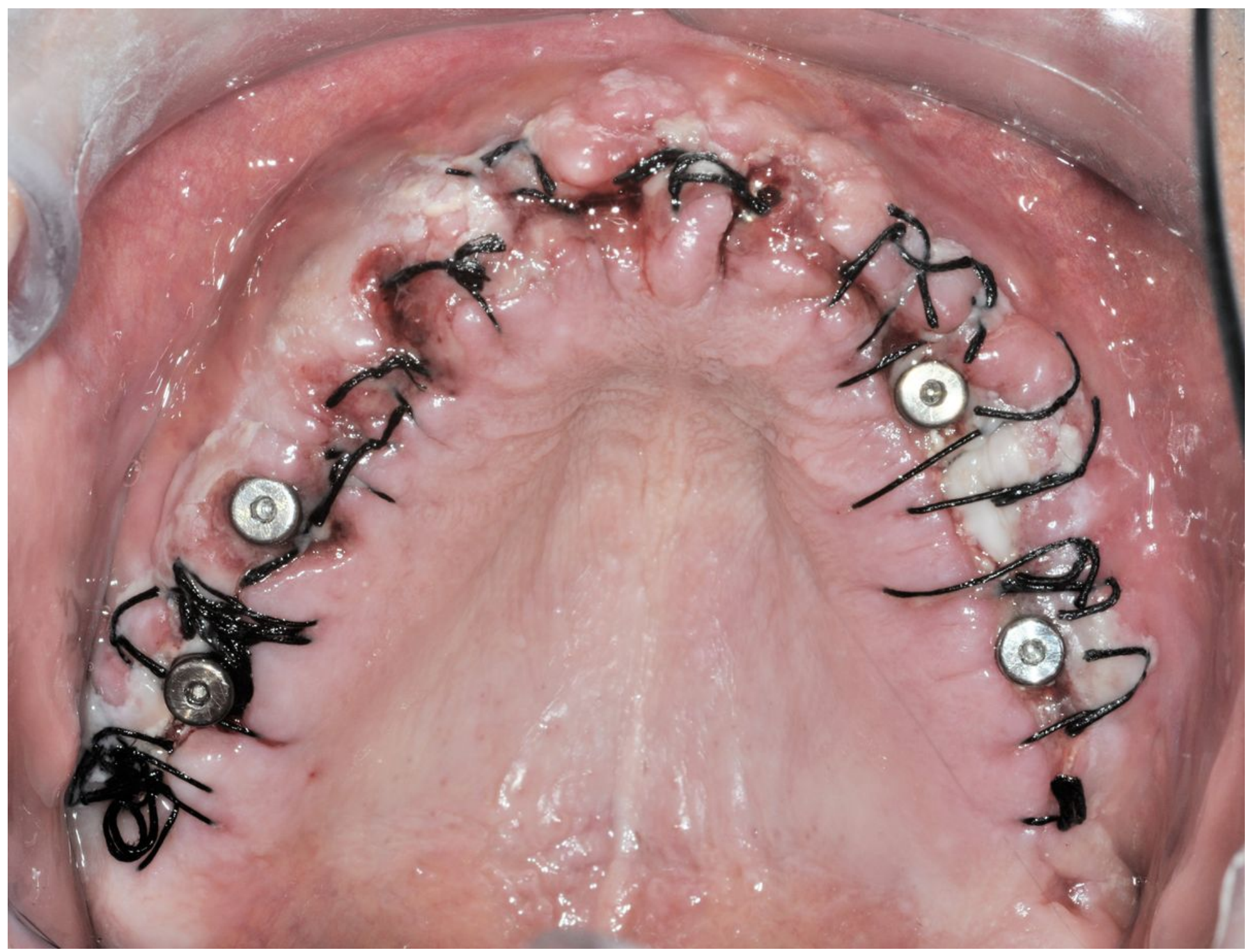

Figure 4

Intra-oral photograph of soft-tissue healing 72 hours after surgery. 


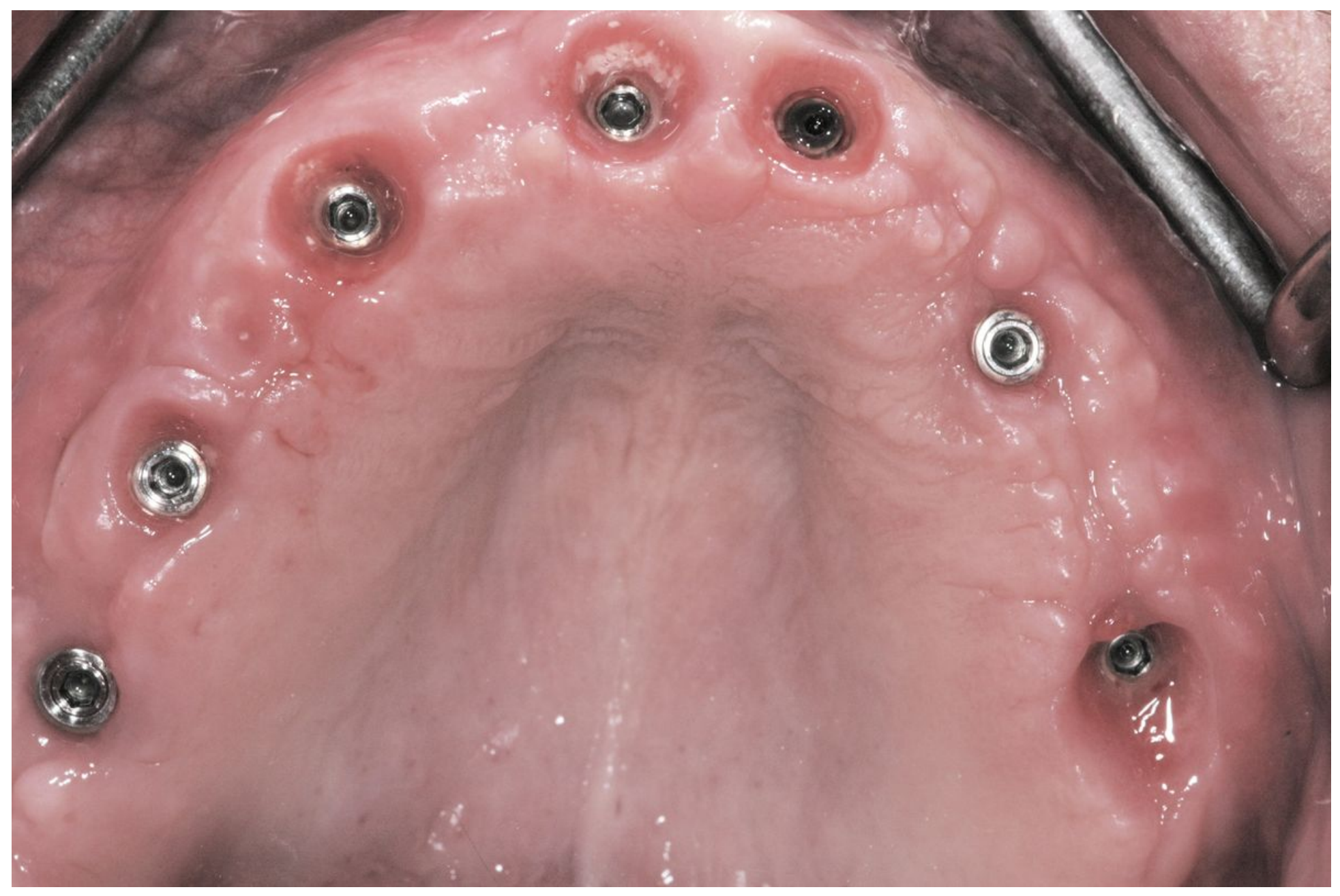

\section{Figure 5}

Intra-oral photograph of soft tissue at a 4-month follow-up visit.

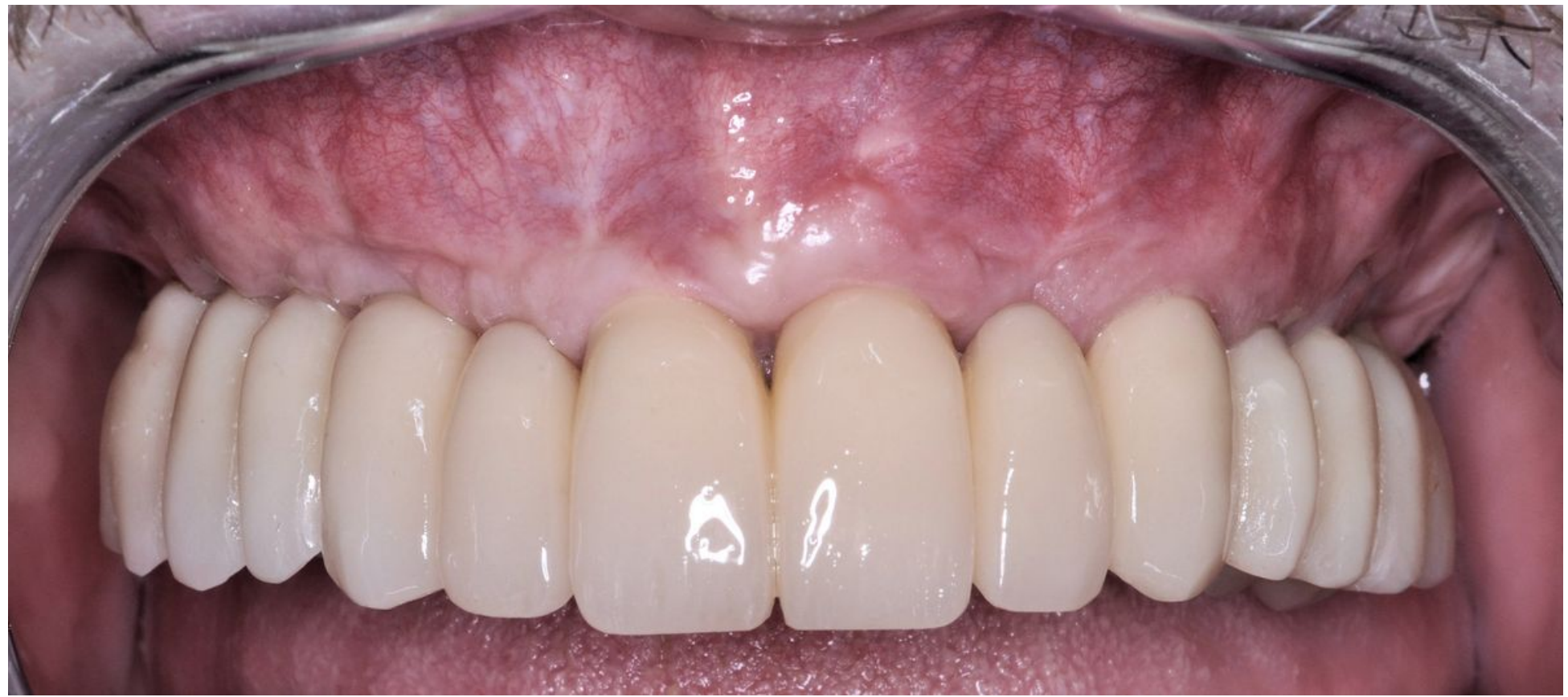

Figure 6 
Intra-oral photograph of the restoration at a 4-month follow-up visit.

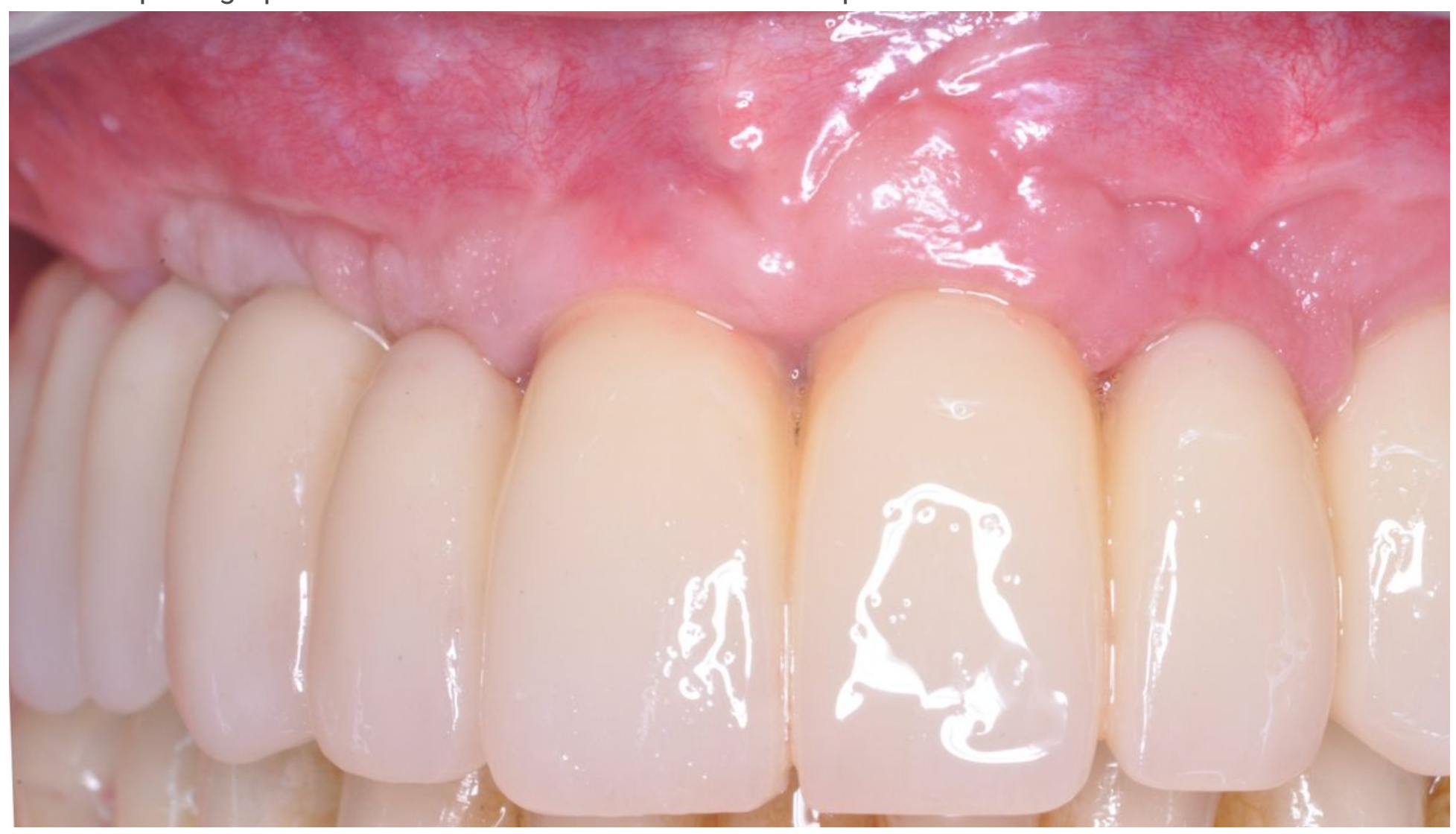

\section{Figure 7}

Intra-oral photograph of the restoration at a 8-year follow-up visit. 


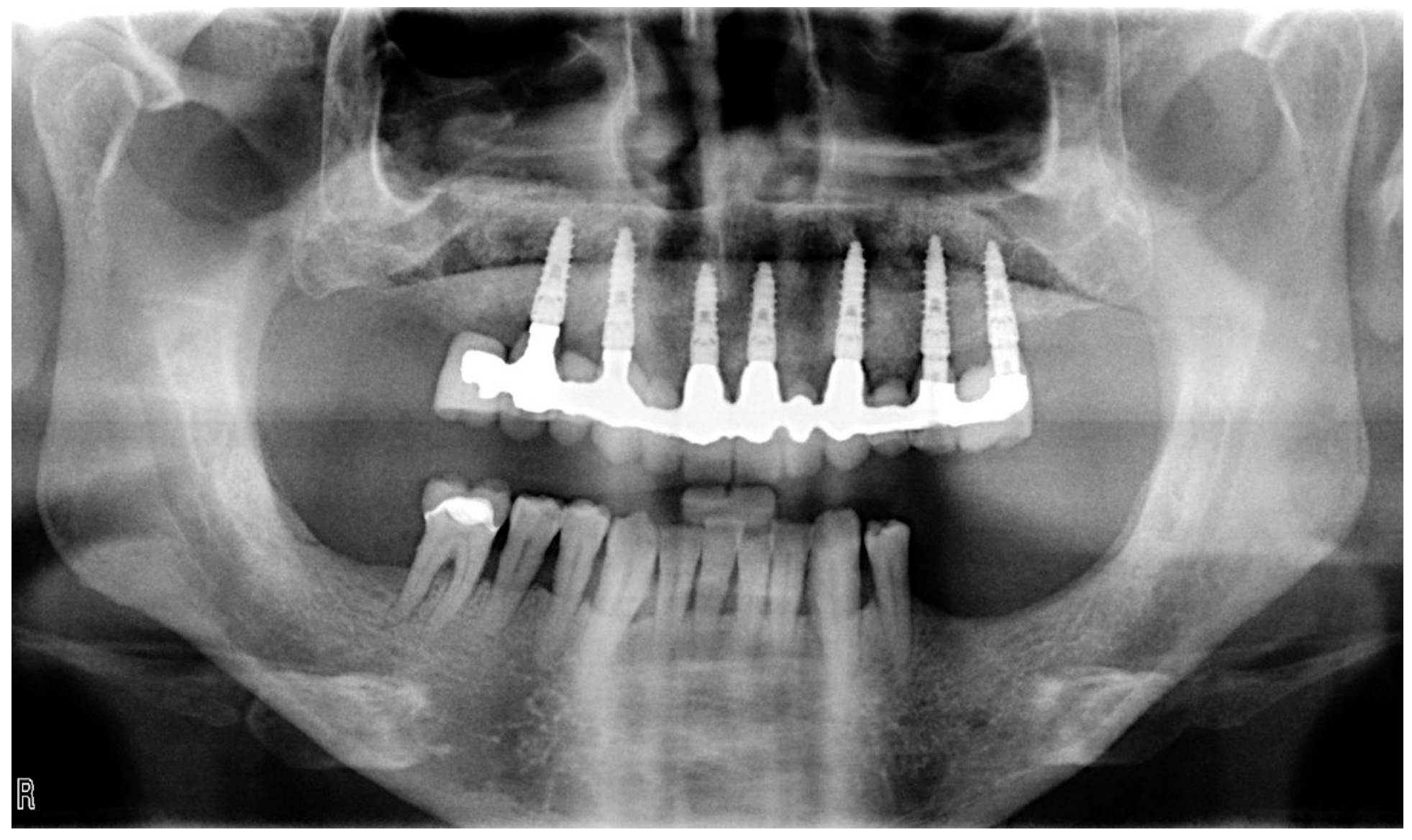

Figure 8

Radiological control at a 8-year follow-up visit.

$$
\text { Strata }+ \text { Smoke }=\text { No }+ \text { Smoke }=\text { Yes }
$$

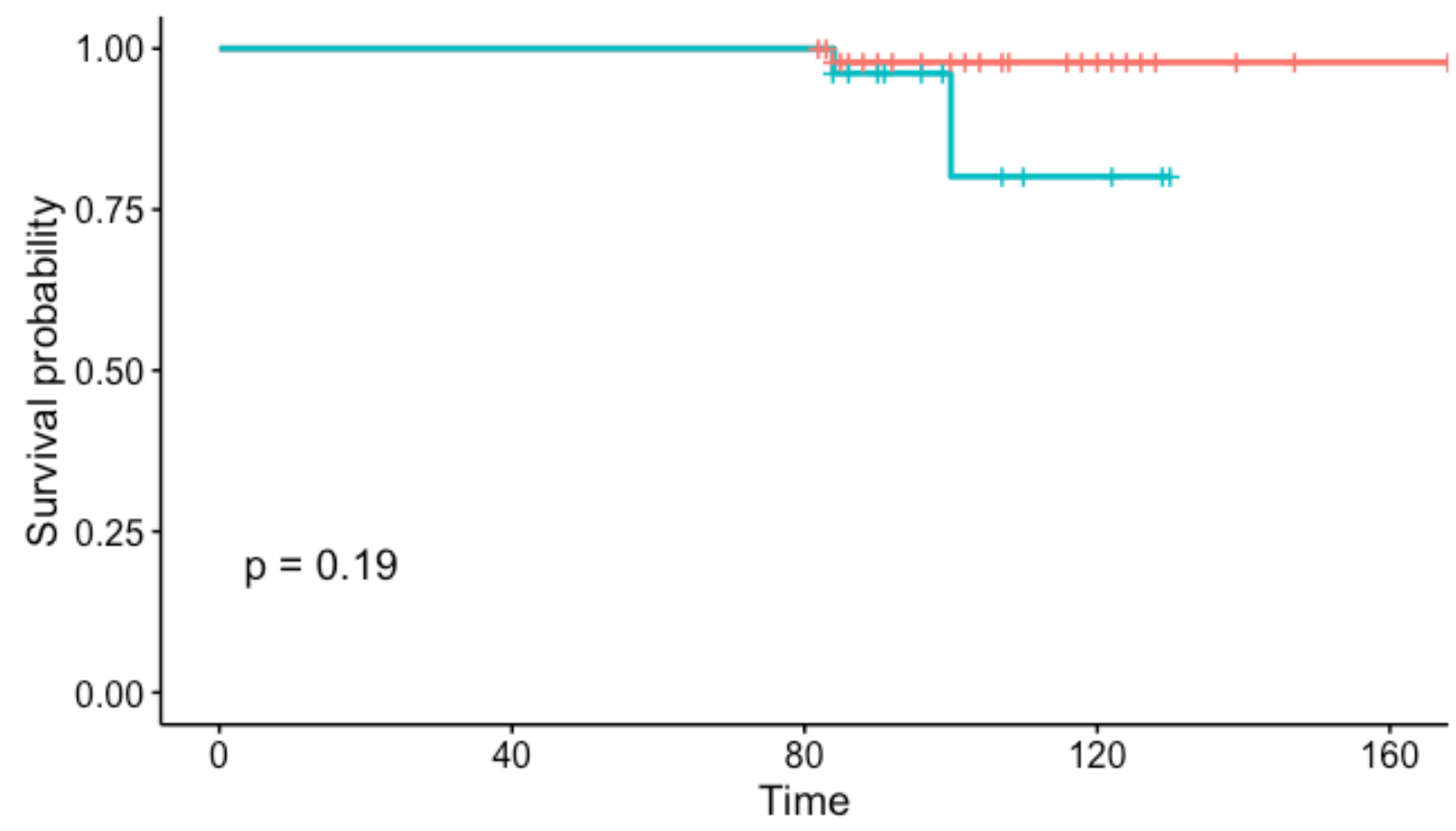




\section{Figure 9}

Graph of Kaplan-Meier cumulative success rate for implants stratified by smoking habit. The verti-cal axis shows the cumulative proportion of non-failed implants.

$$
\text { Strata }+ \text { Jaw=Lower }+ \text { Jaw=Upper }
$$

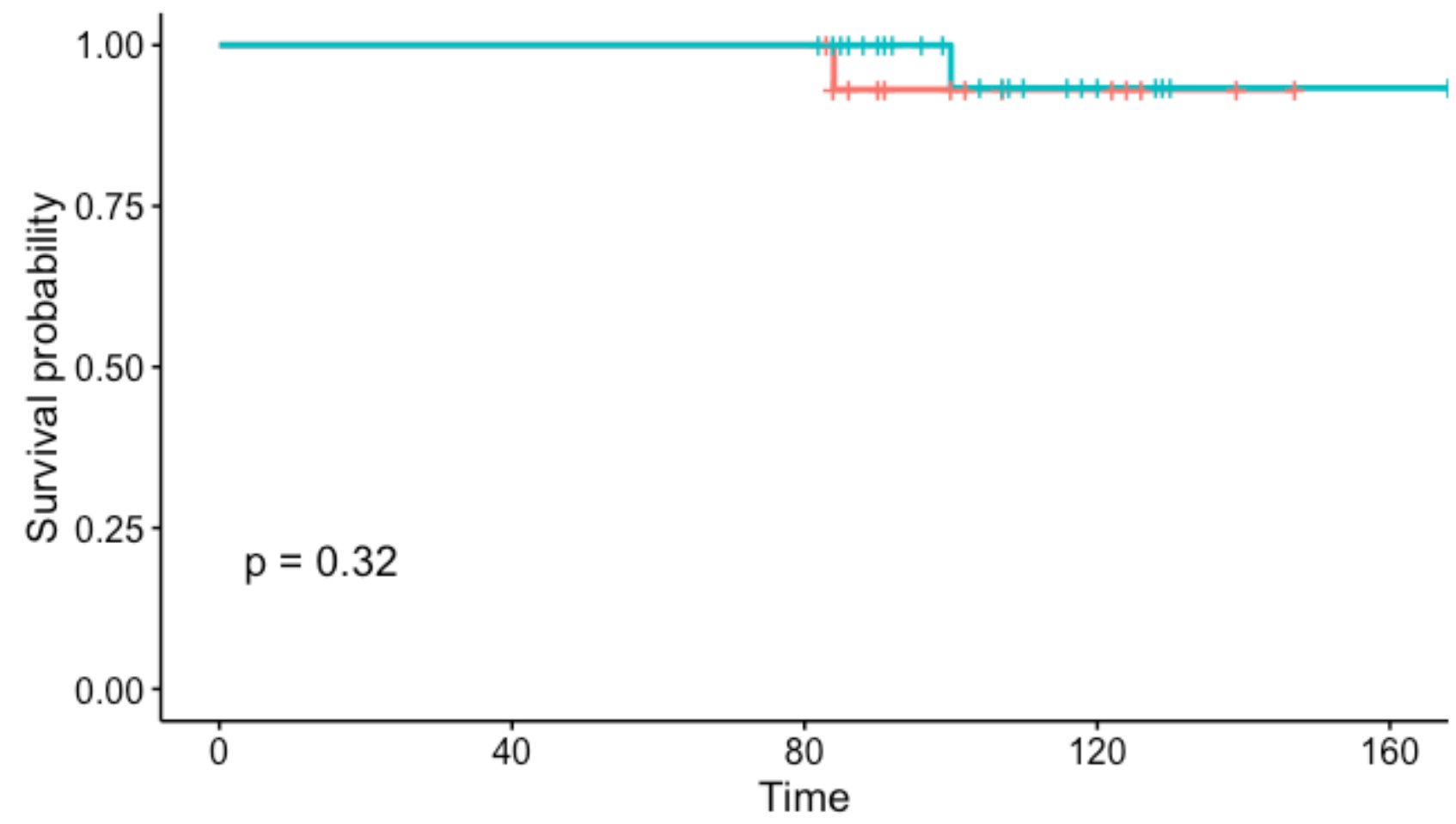

Figure 10

Graph of Kaplan-Meier cumulative success rate for implants stratified by jaw. The vertical axis shows the cumulative proportion of non-failed implants. 


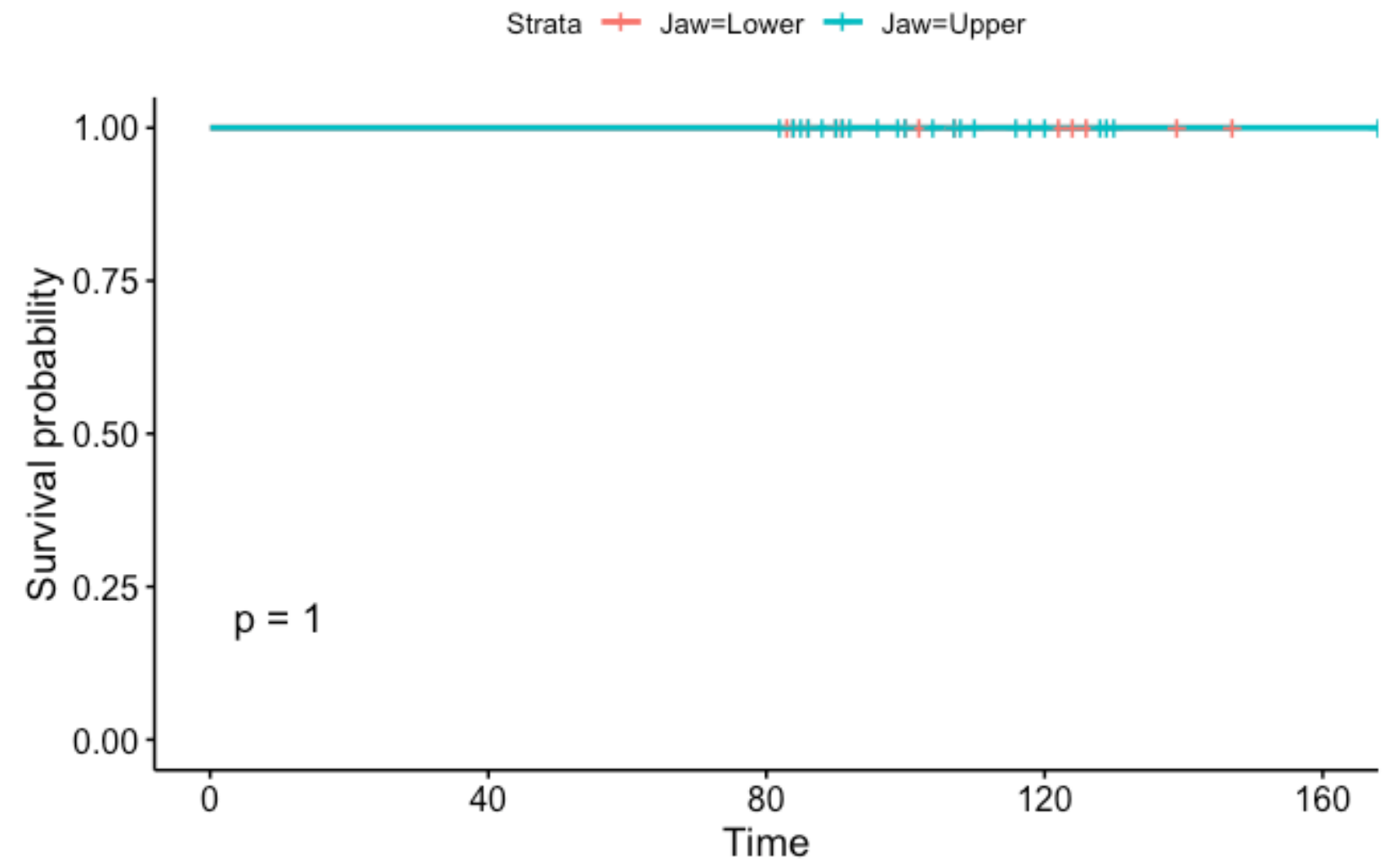

\section{Figure 11}

Graph of Kaplan-Meier cumulative prosthesis survival stratified by jaw. The vertical axis shows the cumulative proportion of non-failed prosthesis. 